\title{
OPEN Antibiofilm and antivirulence efficacy of myrtenol enhances the antibiotic susceptibility of Acinetobacter baumannii
}

\author{
Anthonymuthu Selvaraj ${ }^{1}$, Alaguvel Valliammai ${ }^{1}$, Chandran Sivasankar ${ }^{1,2}$, Manokaran Suba ${ }^{1}$, \\ Ganeshkumar Sakthivel ${ }^{1}$ \& Shunmugiah Karutha Pandian ${ }^{1 \bowtie}$
}

Acinetobacter baumannii $(\mathrm{AB})$ is rising as a human pathogen of critical priority worldwide as it is the leading cause of chronic opportunistic infections in healthcare settings and the condition is ineradicable with antibiotic therapy. $A B$ possesses the ability to form biofilm on abiotic as well as biotic surfaces which plays a major role in its pathogenesis and resistance in clinical settings. Hence, the demand for an alternative therapy to combat the biofilm-associated infections is increasing. The present study explored the antibiofilm potential of myrtenol, a bicyclic monoterpene present in various plants against reference and clinical strains of AB. Myrtenol $(200 \mu \mathrm{g} / \mathrm{mL})$ exhibited a strong antibiofilm activity without exerting any harmful effect on growth and metabolic viability of $A B$ strains. Microscopic analyses confirmed the reduction in the biofilm thickness and surface coverage upon myrtenol treatment. Especially, myrtenol was found to be effective in disrupting the mature biofilms of tested $A B$ strains. Furthermore, myrtenol inhibited the biofilm-associated virulence factors of $A B$ strains such as extracellular polysaccharide, cell surface hydrophobicity, oxidant resistance, swarming and twitching motility. Transcriptional analysis unveiled the suppression of the biofilmassociated genes such as bfmR, csuA/B, bap, ompA, pgaA, pgaC, and katE by myrtenol. Notably, myrtenol improved the susceptibility of $A B$ strains towards conventional antibiotics such as amikacin, ciprofloxacin, gentamicin and trimethoprim. Thus, the present study demonstrates the therapeutic potential of myrtenol against biofilm-associated infections of $A B$.

Acinetobacter baumannii $(\mathrm{AB})$ is a Gram-negative, coccobacillus, opportunistic human pathogen and it most frequently causes nosocomial infections such as ventilator-associated pneumonia, bacteraemia, endocarditis, meningitis, wound infections, respiratory tract and urinary tract infections. It has been listed as one of the pathogens of "ESKAPE" bacteria by Infectious Diseases Society of America (IDSA) because of its resistance to conventional antibiotics ${ }^{1}$. According to the World health organization (WHO) report of 2017, it comes under the critical level in the priority list of multidrug-resistant (MDR) bacteria with extreme threat to human health and it increases the need for the development of new antimicrobial treatments ${ }^{2}$. Resistance capacity of $A B$ is increasing enormously against b-lactams, carbapenems, fluoroquinolones and aminoglycosides worldwide ${ }^{3}$. AB is notorious pathogen due to its ability to produce several virulence factors such as phospholipase, hemolytic activity, siderophore production and acinetobactin synthesis. In addition, $A B$ acquires drug resistance by various mechanisms including quorum sensing, biofilm formation, alteration of outer membrane, secretion systems and efflux pump. Quorum sensing in $\mathrm{AB}$ is a two component regulatory system comprising AbaI/AbaR which is homologous to the prototype LuxI/LuxR system of Gram-negative bacteria. AbaI is a sensor protein encoded by abaI which synthesizes signalling molecules also known as acyl homoserine lactones (AHL) and AbaR is a receptor protein encoded by abaR which binds to the AHL. Once activated by AHL, AbaR induces downstream signalling pathways which control the expression of various virulence factors (ompA, AdeFGH efflux pump, PNAG and type I pili) and biofilm formation. Among various virulence mechanisms, biofilm formation is foremost reason for antibiotic resistance and pathogenesis of $\mathrm{AB}^{4,5}$.

Biofilm is the three dimensional structural community of bacterial cells adhered to each other as well as to substratum by an extracellular matrix containing polysaccharides, proteins and nucleic acids. Biofilm mode 


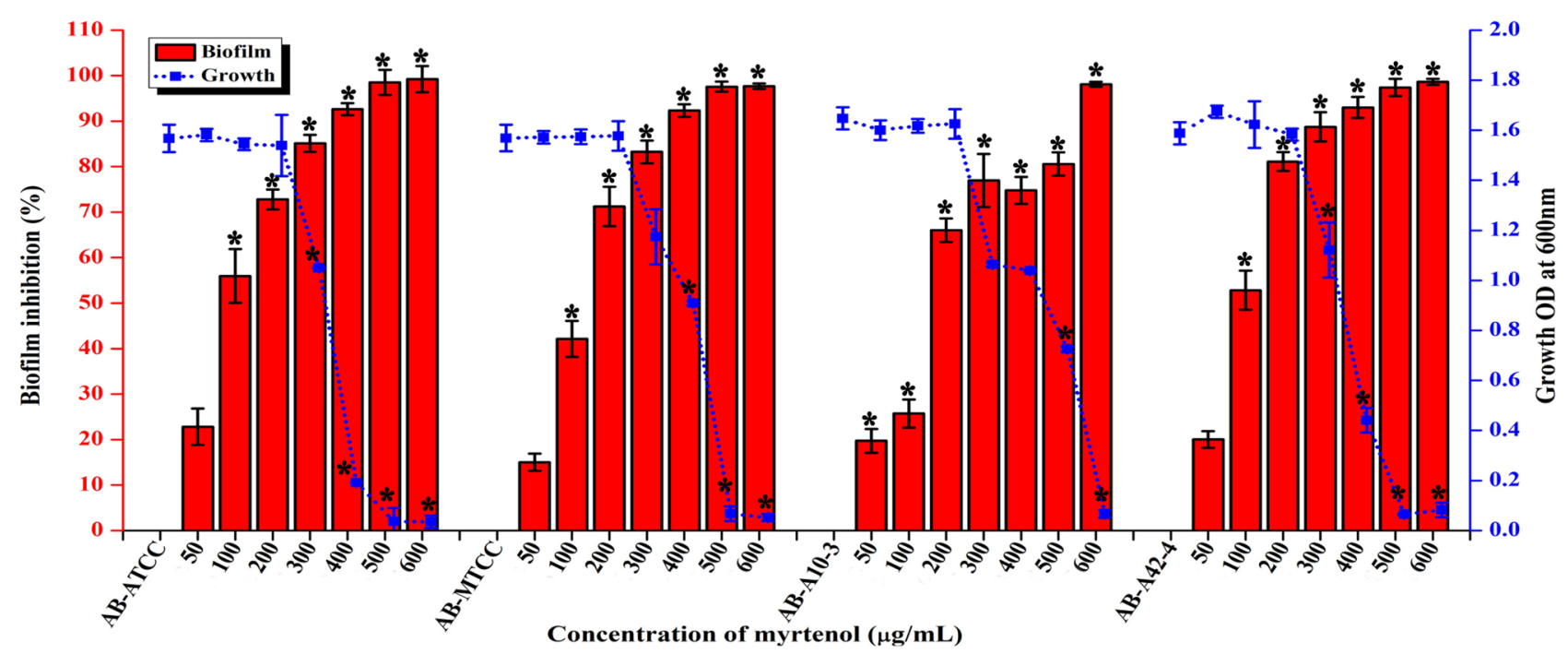

Figure 1. Effect of myrtenol at various concentrations $(50-600 \mu \mathrm{g} / \mathrm{mL})$ on the growth and biofilm formation of AB-ATCC 19606, AB-MTCC 9826, AB-A10-3 and AB-A42-4. Error bars and asterisks indicate the SD and statistical significance $(p<0.05)$, respectively.

of lifestyle increases the resistance to antibiotics and hosts innate immune system, persistent adherence onto biotic surfaces and also the infectious disease-causing ability of bacteria. Furthermore, chronic biofilm causes severe health issues especially in patients with prosthetic medical devices ${ }^{6}$. Furthermore, several virulence factors are associated with $\mathrm{AB}$ biofilms such as biofilm-associated protein (Bap), two-component system (BfmS/ $\mathrm{BfmR}$ ), chaperon-usher pilus (csuA/B, A, B, C, D, and E), poly- $\beta-(1,6)-N$-acetyl glucosamine (PNAG) and outer membrane protein $\mathrm{A}(\mathrm{OmpA})^{7,8}$. Therefore, targeting the biofilm formation of $\mathrm{AB}$ is the effective way to control antibiotic-resistance and biofilm-associated infections. Currently, the need for a novel therapeutic strategy to combat biofilm-associated infection of $\mathrm{AB}$ has gained more attention. Antibiofilm agents attenuate adherence and virulence factors of pathogen instead of affecting its growth and hence the possibility of resistance development is much lower. In addition, antibiofilm therapy enhances the sensitivity of bacteria to antibiotics and the host immune system. This indicates the necessity of antibiofilm therapy and also the importance of the discovery of novel antibiofilm agents ${ }^{9}$. Previously, numerous antibiofilm agents from natural sources have been explored against AB biofilms such as chimeric peptides, curcumin, magainin, honokiol and magnolol ${ }^{10-13}$. Myrtenol (PubChem CID: 10582), a bicyclic Monoterpene, is derived from Myrtus communis, Cyperus rotundus, Tanacetum vulgare, Dendroctonus armandi and Pinus armandii ${ }^{14-17}$. WHO expert committee on food additives recognized myrtenol as a safe flavoring ingredient. It has been well known for its food flavouring potential, fragrance property, non-toxicity and various biological activities such as anti-inflammatory, anti-Alzheimer's, antioxidant, anticancer, gastroprotective activity, anxiolytic, antimicrobial and antibiofilm activity ${ }^{18-22}$. Thus, the present study for the first time evaluated the effect of myrtenol on biofilm associated virulence factors of $A B$.

\section{Results}

Effect of myrtenol on the growth and biofilm formation of AB. To determine the minimum inhibitory concentration (MIC) and minimum biofilm inhibitory concentration (MBIC) of myrtenol against $A B$ strains, microtiter plate method and crystal violet quantification assay were performed, respectively. The result showed that MIC of myrtenol was $500 \mu \mathrm{g} / \mathrm{mL}$ for AB-ATCC 19606, AB-MTCC 9829, AB A10-3 and $600 \mu \mathrm{g} / \mathrm{mL}$ for $\mathrm{AB}$ A42-4 strain (Fig. 1). MBIC can be defined as the minimum concentration of drug that exhibits greater than $50 \%$ of biofilm inhibition without affecting growth. Myrtenol at a concentration of $200 \mu \mathrm{g} / \mathrm{mL}$ showed strong antibiofilm activity against AB-ATCC 19606 (72\%), AB-MTCC 9829 (70\%), AB A10-3 (66\%) and AB A42-4 (80\%) without affecting growth (Fig. 1). Hence, $200 \mu \mathrm{g} / \mathrm{mL}$ was fixed as the MBIC for all tested strains.

Microscopic visualization of AB biofilm. In light microscopic analysis, highly aggregated and wellstructured biofilm formation was observed in $\mathrm{AB}$ control samples whereas in myrtenol treated samples significant reduction in biofilm with dispersed cells was observed in all the tested AB strains (Fig. 2A). To assess the effect of myrtenol on the three dimensional biofilm architecture of $\mathrm{AB}$, confocal laser scanning microscopy (CLSM) analysis was performed. Multi-layered biofilm and high biofilm biomass was observed in control samples. In contrast, great reduction in biofilm biomass, surface covered area and thickness was observed in myrtenol $(200 \mu \mathrm{g} / \mathrm{mL})$ treated AB strains (Fig. 2B). Effect of myrtenol on biofilm thickness, biomass and surface to volume ratio was assessed through Comstat analysis. Results revealed that myrtenol treatment notably affected the tested parameters such as biomass, maximum thickness and surface to volume ratio (Table 1).

Effect of myrtenol on $A B$ ring biofilm formation and adherence on polystyrene surface. $A B$ has ability to form biofilm at air-liquid interfaces by adhering to the top portion of liquid medium. Therefore, 
(A) AB-ATCC
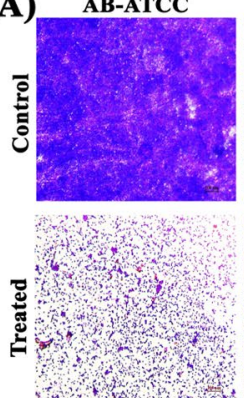

(B)
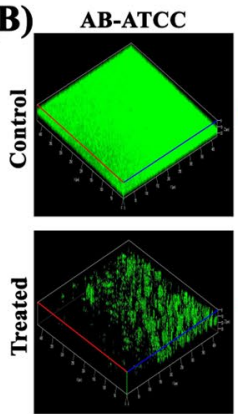

AB-MTCC
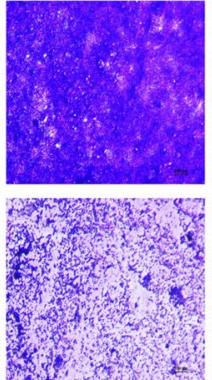

AB-MTCC
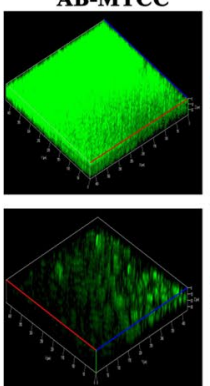

AB-A10-3
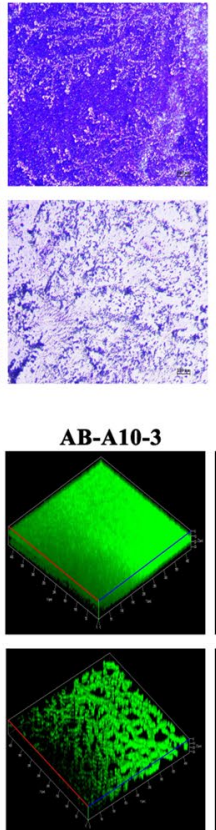

AB-A42-4
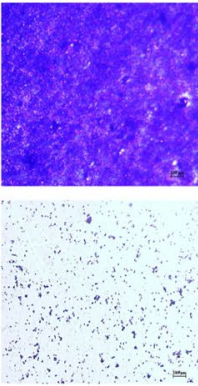

AB-A42-4

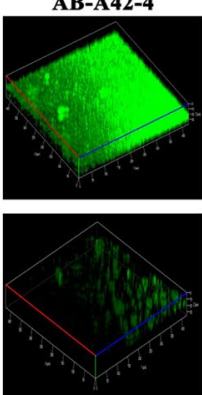

(C)

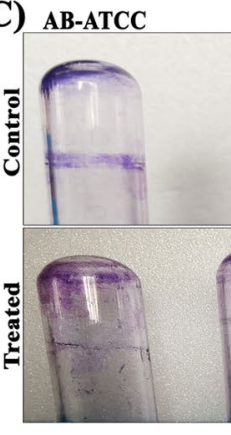

AB-MTCC AB-A42-4

(D)

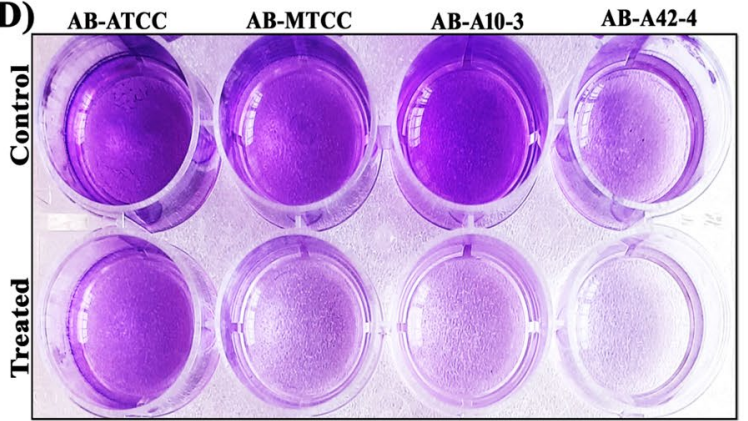

Figure 2. (A) Light and (B) CLSM images representing the antibiofilm efficacy of myrtenol at $200 \mu \mathrm{g} / \mathrm{mL}$ concentration against AB-ATCC 19606, AB-MTCC 9826, AB-A10-3 and AB-A42-4. Inhibitory potential of myrtenol on (C) ring biofilm formation and (D) adherence on polystyrene surface by AB-ATCC 19606, AB-MTCC 9826, AB-A10-3 and AB-A42-4.

\begin{tabular}{|c|c|c|c|c|c|c|}
\hline \multirow[b]{2}{*}{$\mathrm{AB}$ strains } & \multicolumn{2}{|c|}{ Biomass $\left(\mu \mathrm{m}^{3} / \mu \mathrm{m}^{2}\right)$} & \multicolumn{2}{|c|}{ Maximum thickness $(\mu \mathrm{m})$} & \multicolumn{2}{|c|}{$\begin{array}{l}\text { Surface to volume ratio } \\
\left(\mu \mathrm{m}^{2} / \mu \mathrm{m}^{3}\right)\end{array}$} \\
\hline & Control & Treated & Control & Treated & Control & Treated \\
\hline AB-ATCC & $18.68 \pm 0.85$ & $8.64 \pm 1.20$ & $19.90 \pm 0.90$ & $4.87 \pm 0.53$ & $1.05 \pm 0.09$ & $2.15 \pm 0.24$ \\
\hline AB-MTCC & $28.63 \pm 0.90$ & $15.43 \pm 0.92$ & $55.41 \pm 1.07$ & $21.91 \pm 1.38$ & $0.76 \pm 0.04$ & $1.92 \pm 0.15$ \\
\hline AB-A10-3 & $23.18 \pm 0.86$ & $9.67 \pm 1.16$ & $30.00 \pm 1.05$ & $16.80 \pm 1.48$ & $0.53 \pm 0.23$ & $1.95 \pm 0.21$ \\
\hline AB-A42-4 & $24.44 \pm 1.54$ & $12.57 \pm 0.62$ & $36.56 \pm 2.44$ & $19.10 \pm 2.50$ & $0.71 \pm 0.00$ & $1.39 \pm 0.16$ \\
\hline
\end{tabular}

Table 1. Comstat analysis of biofilms formed by AB strains in the absence and presence of myrtenol.

the effect of myrtenol on ring biofilm formation of $\mathrm{AB}$ was assessed. Myrtenol reduced the air-liquid interface biofilm formation of all the tested $\mathrm{AB}$ strains (Fig. 2C). In addition, myrtenol effectively inhibited the attachment of $\mathrm{AB}$ strains on polystyrene surface as exhibited by crystal violet staining (Fig. 2D).

Myrtenol disrupts mature biofilm formed by AB. To check the biofilm disrupting ability of myrtenol, mature biofilm disruption assay was performed. Results revealed the potential of myrtenol in disrupting the preformed biofilms of all the tested $\mathrm{AB}$ strains (Fig. 3).

Non-antibacterial effect of myrtenol on AB. To determine the non-antibacterial effect of myrtenol against both biofilm and planktonic cells of $\mathrm{AB}$ strains, Alamar blue assay was performed. Alamar blue assay revealed that myrtenol does not affect the cell viability of tested $\mathrm{AB}$ strains as results showed no significant variance in the fluorescence intensity of control and myrtenol treated sample (Fig. 4).

Myrtenol affects extracellular polysaccharides (EPS) production and cell surface hydrophobicity $(\mathrm{CSH})$ of $\mathrm{AB}$. The EPS production in $\mathrm{AB}$ is directly associated with biofilm formation and maintains the biofilm integrity. The quantitative mass analysis of EPS production in myrtenol treated samples indicated a significant reduction of EPS. At MBIC $(200 \mu \mathrm{g} / \mathrm{mL})$ of myrtenol, more than $60 \%$ reduction in EPS was observed in all the tested $\mathrm{AB}$ strains when compared to control sample (Fig. 5A). Furthermore, $\mathrm{CSH}$ of $\mathrm{AB}$ is playing significant role in its adherence to both biotic and abiotic surfaces. Hence, the effect of myrtenol on $\mathrm{CSH}$ of $\mathrm{AB}$ was examined and result showed a constant reduction in $\mathrm{CSH}$ of myrtenol treated $\mathrm{AB}$ strains when compared to the control sample (Fig. 5B). This result confirmed that myrtenol affects the $\mathrm{CSH}$ of $\mathrm{AB}$. 


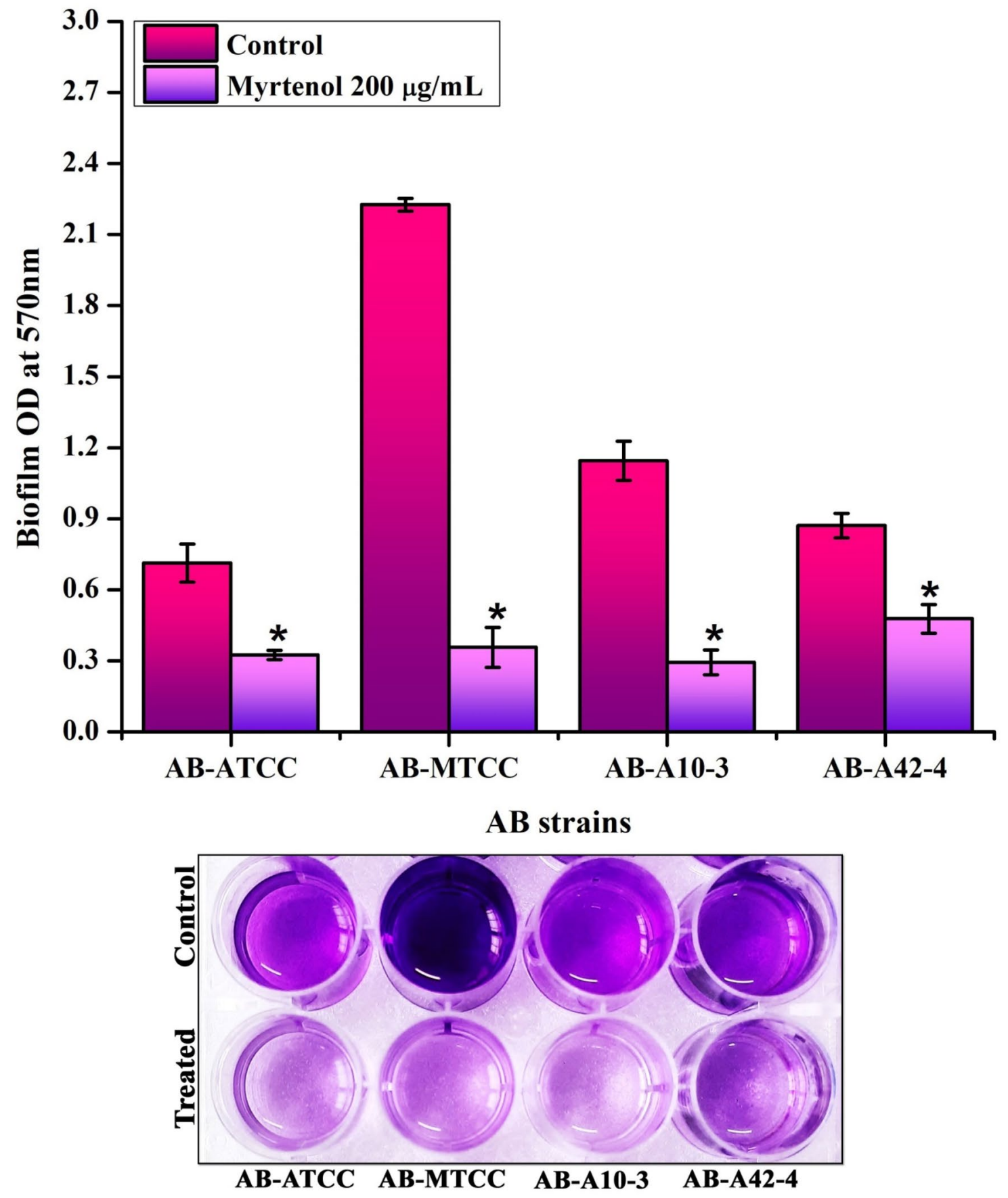

Figure 3. Biofilm disrupting potential of myrtenol $(200 \mu \mathrm{g} / \mathrm{mL})$ on preformed biofilms of AB-ATCC 19606 , AB-MTCC 9826, AB-A10-3 and AB-A42-4. Bottom panel depicts the mature biofilm disruptive potential of myrtenol against $\mathrm{AB}$ strains. Error bars and asterisks indicate the SD and statistical significance $(p<0.05)$, respectively.

Myrtenol impedes the motility of AB. AB is exploiting the flagellum and pili for motility, environmental survival and colonization on different surfaces. Therefore, swarming and twitching motility assays were performed in the absence and presence of myrtenol. Interestingly, myrtenol inhibited the swarming and twitching motility of $\mathrm{AB}$ to a great extent. At $200 \mu \mathrm{g} / \mathrm{mL}$ concentration of myrtenol, maximum reduction in swarming motility of $\mathrm{AB}$ strains was observed compared to high swarming motility of control sample (Fig. 6A). Twitching motility was also found to be inhibited at $200 \mu \mathrm{g} / \mathrm{mL}$ concentration of myrtenol when compared to the control samples (Fig. 6B).

Myrtenol reduces the resistance of AB to oxidants. Reactive oxygen species (ROS) generated by host immune cells are responsible for innate immune clearance of bacterial pathogen. Therefore, hydrogen peroxide $\left(\mathrm{H}_{2} \mathrm{O}_{2}\right)$ sensitivity assay was performed to assess the effect of myrtenol on sensitivity of $\mathrm{AB}$ to ROS. From the 


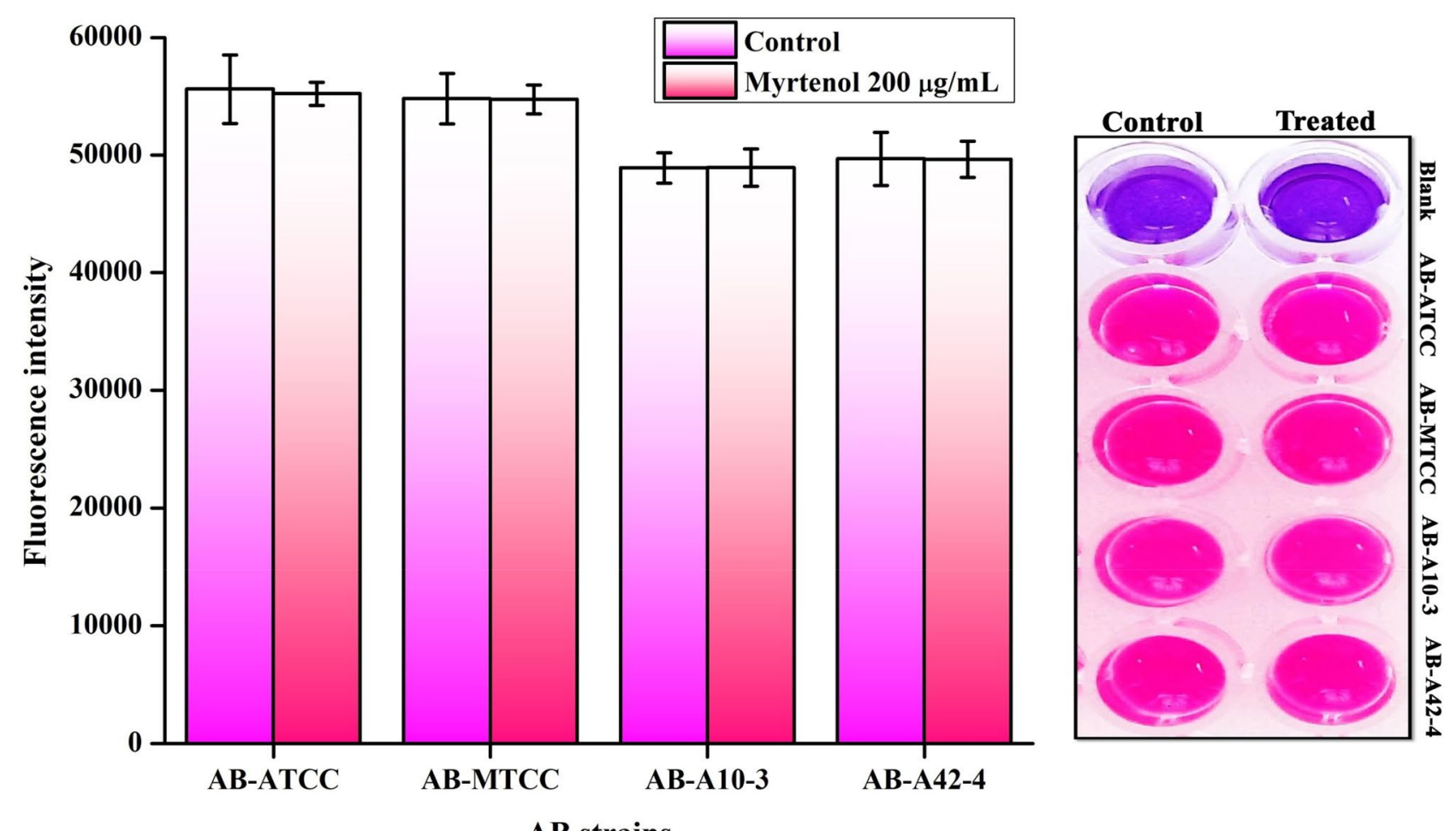

\section{AB strains}

Figure 4. Effect of myrtenol ( $200 \mu \mathrm{g} / \mathrm{mL})$ on viability of AB-ATCC 19606, AB-MTCC 9826, AB-A10-3 and $\mathrm{AB}-\mathrm{A} 42-4$ as assessed by Alamar blue reduction assay. Error bars indicate the SD.
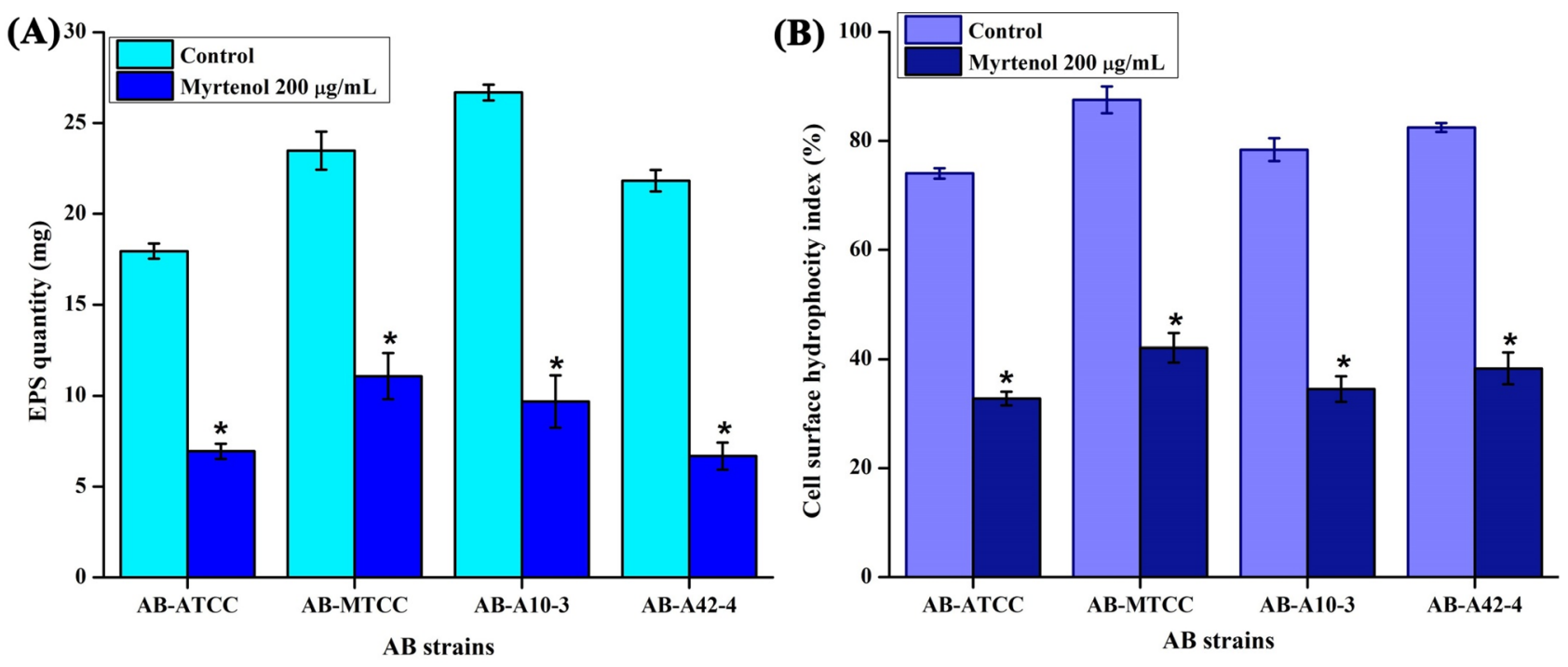

Figure 5. Inhibitory effect of myrtenol $(200 \mu \mathrm{g} / \mathrm{mL})$ on (A) EPS production and (B) CSH of AB-ATCC 19606, AB-MTCC 9826, AB-A10-3 and AB-A42-4. Error bars and asterisks indicate the SD and statistical significance $(p<0.05)$, respectively.

result it was observed that myrtenol at $200 \mu \mathrm{g} / \mathrm{mL}$ significantly reduced the survival of $\mathrm{AB}$ strains when compared to untreated control sample (Fig. 7A). The result of $\mathrm{H}_{2} \mathrm{O}_{2}$ disc diffusion assay also showed the increased zone of clearance in the presence of myrtenol $(200 \mu \mathrm{g} / \mathrm{mL})$ when compared to zone of clearance of $\mathrm{AB}$ control cells. The increasing zone of clearance observed in myrtenol treated samples indicates the enhanced sensitivity of $\mathrm{AB}$ to ROS (Fig. 7B). 

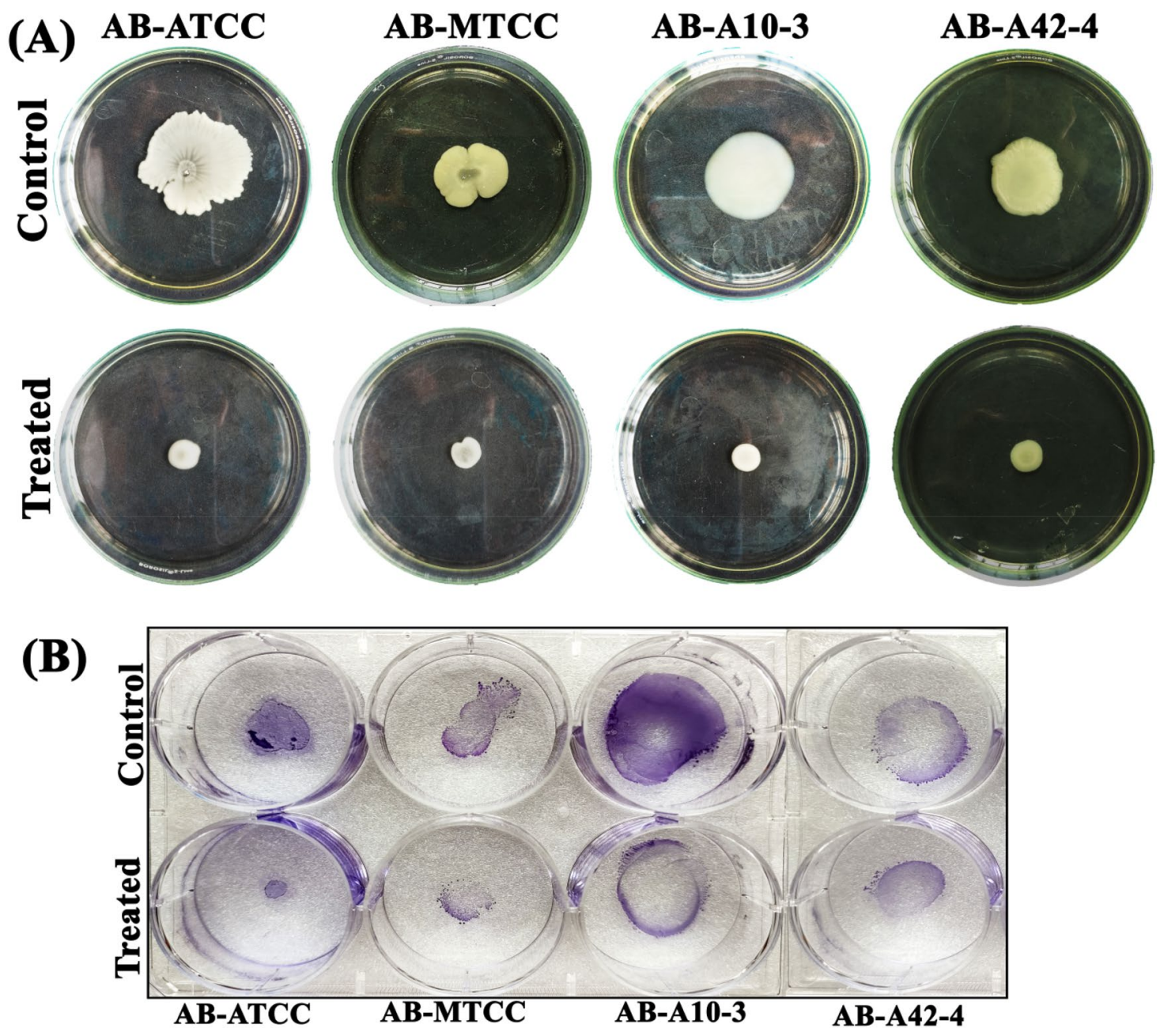

Figure 6. Effect of myrtenol $(200 \mu \mathrm{g} / \mathrm{mL})$ on (A) swarming and (B) twitching motility of AB-ATCC 19606, AB-MTCC 9826, AB-A10-3 and AB-A42-4.
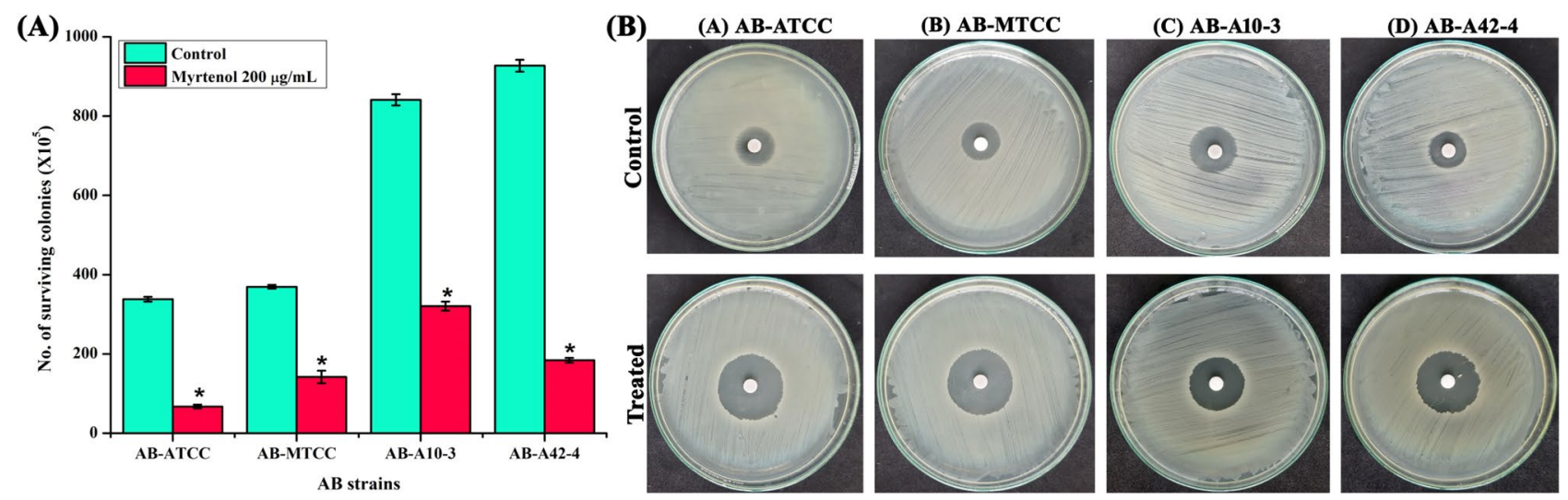

Figure 7. (A) Effect of myrtenol $(200 \mu \mathrm{g} / \mathrm{mL})$ on the survival of AB-ATCC 19606, AB-MTCC 9826, AB-A10-3 and $\mathrm{AB}-\mathrm{A} 42-4$ in the presence of $\mathrm{H}_{2} \mathrm{O}_{2}$ and (B) plate images showing the effect of myrtenol on the sensitivity of $\mathrm{AB}$ strains to $\mathrm{H}_{2} \mathrm{O}_{2}$ by disc diffusion assays. Error bars and asterisks indicate the $\mathrm{SD}$ and statistical significance $(p<0.05)$, respectively. 


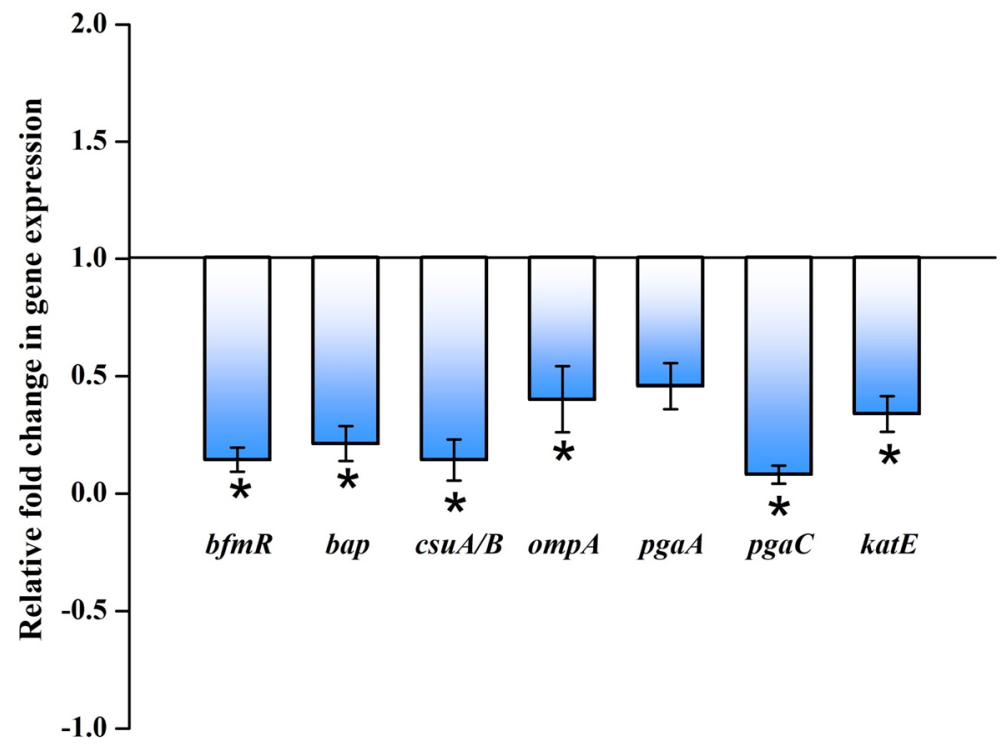

Figure 8. Effect of myrtenol $(200 \mu \mathrm{g} / \mathrm{mL})$ on expression profile of genes involved in biofilm formation in AB-ATCC 19606. Error bars and asterisks indicate the SD and statistical significance $(p<0.05)$, respectively.

Myrtenol modulates the expression of biofilm associated genes in AB. qPCR analysis was performed to assess the effect of myrtenol on expression of biofilm associated genes in $A B$. The result revealed a significant reduction in the expression of $b f m R$, bap, csuA/B, ompA, pgaA, pgaC and katE (Fig. 8).

Myrtenol improves the antibiotic susceptibility of $A B$. The antibiotics susceptibility of $A B$ was assessed in the absence and presence of myrtenol $(200 \mu \mathrm{g} / \mathrm{mL})$ for which initially MIC of antibiotics such as amikacin, ciprofloxacin, gentamicin and trimethoprim was determined by microbroth dilution method (Supplementary Table 1). Interestingly, addition of myrtenol considerably increased the sensitivity of antibiotics against $\mathrm{AB}$ strains (Fig. 9). Further, zone of inhibition of antibiotics was also found to be increased in the presence of myrtenol (Table 2; Supplementary Fig. 1). These results clearly indicate that myrtenol increases the susceptibility of $\mathrm{AB}$ strains towards the antibiotics.

\section{Discussion}

The biofilm formation enhances the infectious disease-causing ability of $\mathrm{AB}$ in healthcare settings and is also responsible for the emergence of antibiotic resistance. Thus, antibiofilm based approach to fight infectious diseases has gained more attention among the scientific community. Myrtenol is a non-toxic natural molecule and though well known for various biological activities ${ }^{18-21}$, antibiofilm efficacy of myrtenol against $\mathrm{AB}$ remains unexplored. In the present study, antibiofilm potential of myrtenol was assessed against two reference strains (AB-ATCC 19606 and AB-MTCC 9829) and two clinical isolates (AB A10-3 and AB A42-4). Crystal violet quantification assay revealed that myrtenol at $200 \mu \mathrm{g} / \mathrm{mL}$ concentration could significantly inhibit biofilm formation of AB-ATCC 19606, AB-MTCC 9829, AB A10-3 and AB A42-4 strains by $72 \%, 70 \%, 65 \%$ and $80 \%$, respectively. In order to examine the effect of myrtenol on biofilm architecture, light microscopic, CLSM and Comstat analyses were performed and results depicted the substantial reduction in the biofilm covered surface area as well as the thickness of biofilm architecture in myrtenol treated samples compared to the control, where multilayered biofilm was observed in high quantity. In addition, myrtenol was able to inhibit biofilm formation of $A B$ strains on glass and polystyrene surfaces. Most notably, myrtenol has the potential to disrupt preformed biofilms of $\mathrm{AB}$ strains which is very appreciable nature of myrtenol as mature biofilms of $\mathrm{AB}$ formed in clinical context is very challenging to eradicate and is responsible for persistence of infection ${ }^{21}$. An ideal antibiofilm agent is expected to have non-fatal effect on bacterial growth and hence the chance of causing any selective pressure on the bacteria is much lower. Consequently, the results of Alamar blue assay unveiled the non-bactericidal effect of myrtenol on $\mathrm{AB}$ and non-bactericidal antibiofilm efficacy of myrtenol is highly beneficial in therapeutic aspect. The bacterial biofilm is made up with EPS which acts as barrier to antibiotics and host immune system. Several studies have revealed the important role of EPS in biofilm formation such as protection from environmental stress, providing stability to biofilm matrices and enhanced cell to cell adherence. Thus, inhibiting the production of EPS could increase the sensitivity of bacteria to antibiotics and host innate immune system and eventually it helps to recover biofilm-associated infections ${ }^{23-25}$. Hence, the EPS was extracted from control and myrtenol treated cultures and the results indicated the EPS inhibitory effect of myrtenol. The EPS production influences the CSH of bacteria thereby it enhances bacterial colonization and attachment to biotic and abiotic surfaces. Prior research has shown that the distribution of EPS affects the CSH wherein the quantity of EPS production was directly linked with CSH of cells ${ }^{26}$. Thus, MATH assay was done to assess the effect of EPS reduction on CSH of control and myrtenol treated cells. The result of MATH assay showed significant inhibition in the CSH of 
(A)

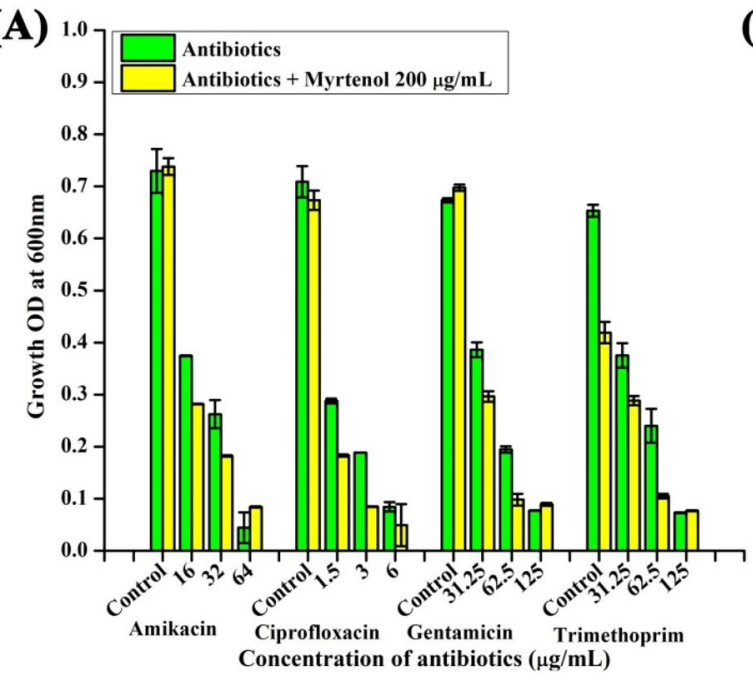

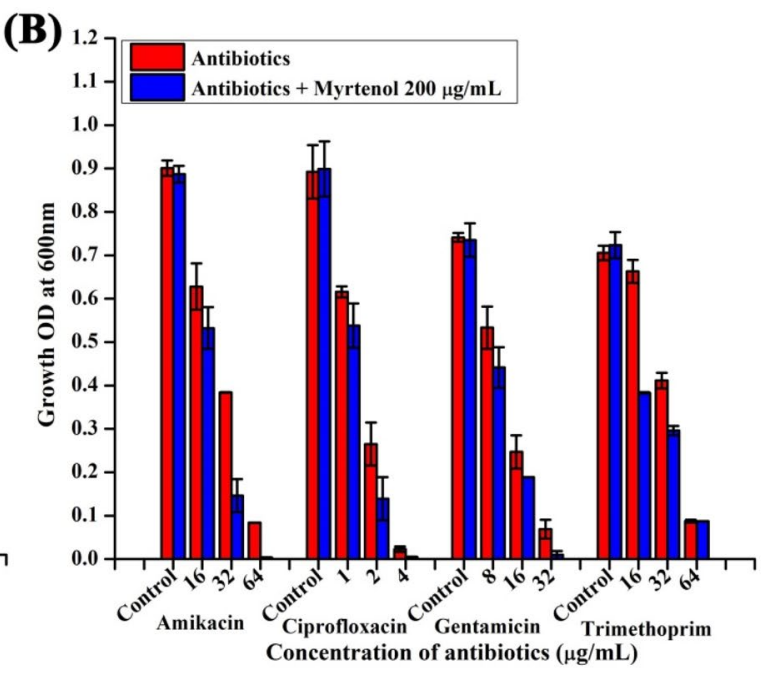
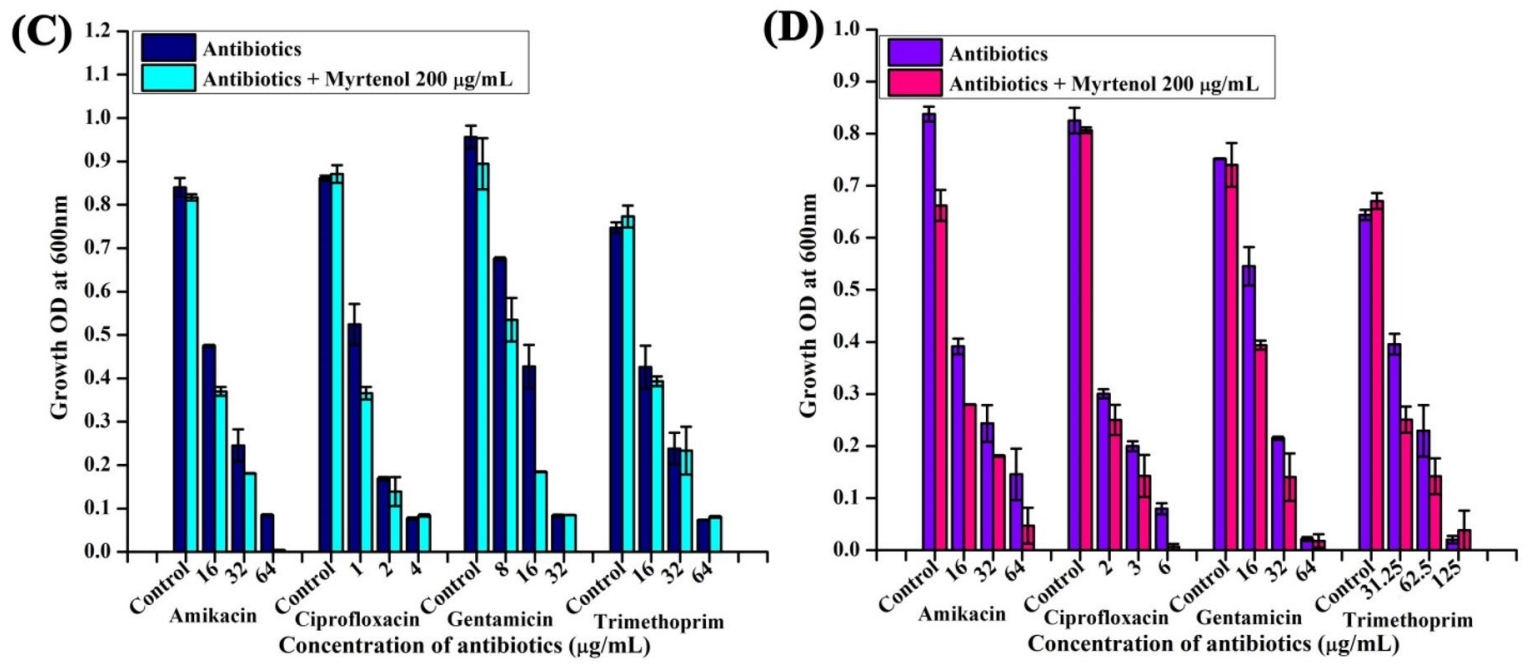

Figure 9. Effect of myrtenol $(200 \mu \mathrm{g} / \mathrm{mL})$ on the susceptibility of AB-ATCC 19606, AB-MTCC 9826, AB-A10-3 and AB-A42-4 to antibiotics. Error bars and asterisks indicate the SD and statistical significance $(p<0.05)$, respectively.

\begin{tabular}{|l|c|l|l|l|l|l|l|l|}
\hline & \multicolumn{3}{|c|}{ AB-ATCC } & AB-MTCC & \multicolumn{2}{l|}{ AB-A10-3 } & \multicolumn{2}{l|}{ AB-A42-4 } \\
\cline { 2 - 9 } & Control & Treated & Control & Treated & Control & Treated & Control & Treated \\
\hline Amikacin & $9 \pm 0$ & $11.83 \pm 0.29$ & $13.50 \pm 0.50$ & $15.33 \pm 0.58$ & $14.67 \pm 0.58$ & $17.17 \pm 0.29$ & $11.33 \pm 0.58$ & $14.0 \pm 0$ \\
\hline Ciprofloxacin & $15.83 \pm 0.29$ & $16.67 \pm 0.58$ & $17.0 \pm 0$ & $18.83 \pm 0.29$ & $17.67 \pm 0.58$ & $18.83 \pm 0.76$ & $13.83 \pm 0.29$ & $18.17 \pm 0.29$ \\
\hline Gentamycin & $13 \pm 0.29$ & $14 \pm 0$ & $14.50 \pm 0.50$ & $18.50 \pm 0.87$ & $14.67 \pm 0.58$ & $16.83 \pm 0.76$ & $10.33 \pm 0.58$ & $12.33 \pm 0.58$ \\
\hline Trimethoprim & $20.0 \pm 0$ & $21.33 \pm 0.29$ & $14.83 \pm 0.29$ & $20.50 \pm 0.50$ & $21.33 \pm 0.58$ & $22.17 \pm 0.29$ & $17.33 \pm 0.58$ & $18.0 \pm 0$ \\
\hline
\end{tabular}

Table 2. Antibiotic sensitivity pattern of $A B$ strains in the absence and the presence of myrtenol.

myrtenol treated sample which can be corroborated with the above theory and as well as another report ${ }^{27}$. The presence of flagella, pili and fimbriae in $\mathrm{AB}$ promotes the bacterial motility and biofilm formation. Flagella and pili are involved in swarming motility and twitching motility, respectively. The fimbriae of $\mathrm{AB}$ also helps bacterial motility thereby it enhances the biofilm formation between air-liquid interfaces. AB motility mechanisms employ the initial attachment of pathogen to host cells and support internal colonization during pathogenesis. This kind of adherence potential of $\mathrm{AB}$ enhances its survival against conventional antimicrobial agents and the human host immune system ${ }^{28-30}$. Previous study showed that the mutation in a gene ( $\left.p i l T\right)$ responsible for motility affects biofilm formation and bacterial adherence ${ }^{31}$. Therefore, we assessed the influence of myrtenol on the motility of $\mathrm{AB}$ and results of motility assays revealed that myrtenol could profoundly affect both the flagella mediated swarming motility and pili associated twitching motility. $\mathrm{AB}$ also forms biofilm on the air-liquid interface and it is also associated with the motility and adherence of $\mathrm{AB}^{32}$. As motility of $\mathrm{AB}$ was found to be affected in the 


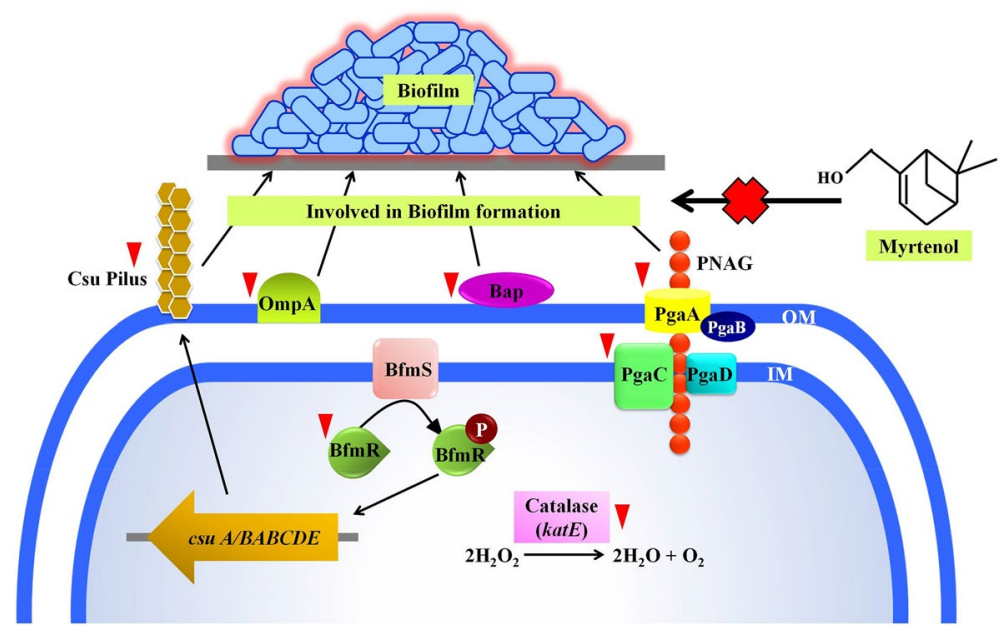

Figure 10. A schematic diagram representing the effect of myrtenol on biofilm associated genes expression in $\mathrm{AB}$. Red coloured inverted triangles indicate down regulation of genes by myrtenol.

presence of myrtenol, the air-liquid interface biofilm formation of $\mathrm{AB}$ was also expected to get affected. In order to validate this, ring biofilm formation assay was performed and as expected, concentration dependent inhibitory activity of myrtenol on air-liquid interface biofilm formation of $\mathrm{AB}$ was observed. In addition, $\mathrm{AB}$ has the ability to synthesize antioxidant enzymes such as catalase and superoxide dismutase to neutralize its own reactive oxygen species (ROS) produced during metabolism and ROS generated in the host during phagocytosis. Antioxidant enzyme production in $\mathrm{AB}$ is connected with the quorum sensing mediated biofilm formation ${ }^{33}$. Thus, the sensitivity of $\mathrm{AB}$ to $\mathrm{H}_{2} \mathrm{O}_{2}$ was analyzed and the obtained results showed that myrtenol treated cells were more susceptible to $\mathrm{H}_{2} \mathrm{O}_{2}$ compared to the control cells. In order to validate the results of phenotypic assays and to identify the mode of antibiofilm activity of myrtenol, qPCR analysis was performed for biofilm-associated genes of $\mathrm{AB}$ such as $b f m R, b a p, c s u A / B$, omp $A, p g a A, p g a C$ and $k a t E^{34,35}$. In $\mathrm{AB}, \mathrm{BfmR}$ is a two-component signal transduction system and it also acts as the master regulator of biofilm initiation. In addition, $B f m R$ regulates the expression of the chaperone-usher system $(c s u A / B A B C D E)$ which promotes pili assembly and fimbriae based bacterial motility ${ }^{36,37}$. The qPCR data showed down-regulation of $b f m R$ and $c s u A$ and it was in total agreement with the result of motility and ring biofilm formation assays. Bap of $\mathrm{AB}$ is one of the cell surface-associated proteins and involved in biofilm maintenance and maturation. Mutation in the bap gene showed the inhibition in the biofilm formation by $\mathrm{AB}^{38,39}$. OmpA of $\mathrm{AB}$ is associated with several biological processes such as bacterial adherence to different surfaces and antimicrobial resistance. Previous study demonstrated the key role of OmpA on bacterial adherence to human keratinocyte and bronchial epithelial cells ${ }^{40}$. In this study, the results of qPCR analysis showed the down-regulation of ompA and bap genes which can be connected with results of biofilm quantification and MATH assays. pgaABCD locus of $A B$ produces the proteins required for the synthesis of PNAG which is a major polysaccharide component of the biofilm matrix. According to a previous report, the deletion of the pga locus strongly affects the biofilm formation in $\mathrm{AB}^{41}$. Thus, the effect of myrtenol on expression of $p g a A$ and $p g a C$ genes was evaluated through qPCR analysis and result showed down-regulation of $p g a A$ and $p g a C$ genes and it is in line with the results of EPS quantification assay. The result obtained from the $\mathrm{H}_{2} \mathrm{O}_{2}$ sensitivity assay showed that the resistance of $\mathrm{AB}$ to oxidants has been reduced upon myrtenol treatment. Hence, the effect of myrtenol on the expression of the $k a t E$ gene which is responsible for catalase production was tested and it comes under the QS mediated biofilm formation system of $\mathrm{AB}$. The down-regulation of the katE gene by myrtenol could be attributed to the improved susceptibility of $\mathrm{AB}$ to $\mathrm{H}_{2} \mathrm{O}_{2}$ in the presence of myrtenol ${ }^{42,43}$. On the whole, myrtenol effectively inhibited the biofilm and virulence of $\mathrm{AB}$ and the schematic representation of myrtenol targeted virulence factors of $\mathrm{AB}$ is depicted in Fig. 10. Combinatorial drug therapy is an emerging strategy to prevent bacterial infections. It has an advantage of decreasing the drug dosage level thereby it reduces drug toxicity and development ofantibacterial resistance. Previous studies reported that antibiofilm agents increased the sensitivity of chronic infection-causing bacteria towards antibiotics ${ }^{44,45}$. Hence, the influence of myrtenol treatment on $\mathrm{AB}$ sensitivity to antibiotics was evaluated and interestingly myrtenol treatment potentiated the susceptibility of $\mathrm{AB}$ to conventional antibiotics namely amikacin, ciprofloxacin, gentamicin and trimethoprim. Ability of myrtenol to enhance the antibacterial activity of commonly used antibiotics is highly appreciable in the clinical context.

\section{Conclusion}

Altogether, the present study for the first time revealed the antibiofilm efficacy of myrtenol against $A B$ reference strains and clinical isolates without affecting the growth. Thus the possibility of drug resistance against myrtenol will be much lower. Apart from inhibiting the biofilm of $\mathrm{AB}$, myrtenol also disrupted the preformed biofilms of $\mathrm{AB}$ strains. In addition, myrtenol affected the biofilm-associated adherence factors such as EPS, CSH, swarming and twitching motility as revealed by in vitro assays. Further, qPCR analysis exhibited that myrtenol suppressed the expression of key genes involved in biofilm formation. Especially, the antibiofilm potential of myrtenol 
enhanced the susceptibility of $\mathrm{AB}$ to oxidants and conventional antibiotics. Considering antibiofilm and antibiotic combination effect of myrtenol on $\mathrm{AB}$, it could be a promising therapeutic agent against virulence factors of $\mathrm{AB}$.

\section{Methods}

Bacterial strains and culture conditions. The bacterial strains used in this study are as follows: $\mathrm{AB}$ reference strain ATCC-19606, AB reference strain MTCC-9826, AB clinical isolate A10-3 (GenBank ID: KM099445) and AB clinical isolate A42-4 (GenBank ID: KM099485). Bacterial strains were cultured in tryptic soy broth (TSB) supplemented with $1 \%$ sucrose and $0.5 \%$ yeast extract (TSBSY) to promote biofilm formation and incubated at $37^{\circ} \mathrm{C}$ for $24 \mathrm{~h}$ in an orbital shaker $(160 \mathrm{rpm})$.

Phytochemical. Myrtenol was purchased from Sigma-Aldrich, India and prepared as a $10 \mathrm{mg} / \mathrm{mL}$ in methanol for all the experiments. Methanol was included as negative control in all the assays carried out.

Determination of MIC. MIC of myrtenol against AB was determined using microtiter plate method. A 96-well plate was filled with $200 \mu \mathrm{L}$ of TSBSY containing one percent of overnight culture of $\mathrm{AB}$ ( $10^{8}$ cells) and increasing concentrations of myrtenol $(50,100,200,400,500$ and $600 \mu \mathrm{g} / \mathrm{mL})$ and incubated at $37^{\circ} \mathrm{C}$ for $24 \mathrm{~h}$. After incubation, the cell density of the culture was measured at $600 \mathrm{~nm}$ using Spectramax M3, Molecular devices, United States ${ }^{46}$.

Determination of MBIC. One percent overnight culture of $\mathrm{AB}$ was used to inoculate $1 \mathrm{~mL}$ of TSBSY in 24-well plate without and with myrtenol and incubated at $37^{\circ} \mathrm{C}$ for $24 \mathrm{~h}$. After incubation, the cell density was measured at $600 \mathrm{~nm}$. After that, planktonic cells were discarded and the well plate containing biofilm cells was washed using sterile phosphate buffered saline (PBS) to remove unbound cells followed by air drying. The plate was then stained with $0.4 \%$ of crystal violet solution for $10 \mathrm{~min}$ and washed with PBS to remove excess stain. The plate was destained by $30 \%$ glacial acetic acid solution and observed at $570 \mathrm{~nm}$. Finally, the percentage of biofilm inhibition was calculated using following formula: $(\%)=\left[\left(\right.\right.$ Control $\mathrm{OD}_{570 \mathrm{~nm}}-$ Treated $\left.\mathrm{OD}_{570 \mathrm{~nm}}\right) /$ Control $\left.\mathrm{OD}_{570 \mathrm{~nm}}\right] \times 100^{47}$.

Air-liquid interface biofilm formation assay. The influence of myrtenol on biofilm formation of $A B$ strains at air-liquid interface was assessed by previously described method. Briefly, overnight culture was added to $2 \mathrm{~mL}$ of TSBSY medium without and with myrtenol at increasing concentrations $(200 \mu \mathrm{g} / \mathrm{mL})$ in the glass test tubes and incubated for $48 \mathrm{~h}$ at $37^{\circ} \mathrm{C}$. After incubation, test tubes were washed thrice with PBS and stained with $0.4 \%$ crystal violet solution ${ }^{48}$.

Microscopic analyses. Biofilm assay was performed on 24-well plate containing glass slides in the absence and presence of myrtenol for $24 \mathrm{~h}$ at $37^{\circ} \mathrm{C}$. After that, the glass slides were washed by sterile PBS and allowed to air dry. For light microscopic analysis, the slides were stained with $0.4 \%$ crystal violet solution and observed at the magnification of $400 \times$ under Nikon Eclipse Ti-S, Tokyo, Japan. For CLSM analysis, acridine orange solution $(0.1 \%)$ was used to stain the glass slides and observed at the magnification of 200× under Zeiss LSM-710, Carl Zeiss, Oberkochen, Germany. Comstat analysis was performed using Comstat 2 software to analyse the biofilm thickness, biomass and surface to volume ratio of biofilm of $\mathrm{AB}$ strains grown in the absence and presence of myrtenol $^{49,50}$.

Biofilm disruption assay. To assess biofilm disruption by myrtenol, preformed $\mathrm{AB}$ biofilms were treated with myrtenol. Briefly, $1 \%$ of AB strains were added to wells of MTP containing $1 \mathrm{~mL}$ of TSBS and incubated for $24 \mathrm{~h}$ at $37^{\circ} \mathrm{C}$. After incubation, medium containing planktonic cells was removed and replaced with fresh TSBS with myrtenol $(200 \mu \mathrm{g} / \mathrm{mL})$ and kept at $37^{\circ} \mathrm{C}$. After $24 \mathrm{~h}$, TSBS was removed and biofilm cells were washed with sterile PBS. Percentage of biofilm disruption was calculated after crystal violet staining as mentioned ${ }^{21}$.

Cell viability assay. Alamar blue assay was performed to assess the influence of myrtenol on viability of AB strains. Briefly, AB strains were grown in 24 well MTP containing $1 \mathrm{~mL}$ of TSBSY medium without and with myrtenol $(200 \mu \mathrm{g} / \mathrm{mL})$ for $24 \mathrm{~h}$ at $37^{\circ} \mathrm{C}$. Then, biofilm formed on polystyrene surface was scraped off and included with planktonic cells for centrifugation at $8000 \mathrm{rpm}$ for $10 \mathrm{~min}$. Then, cell pellets were harvested, washed thrice and resuspended in $1 \mathrm{~mL}$ of PBS. To the cell suspension $(900 \mu \mathrm{L}), 100 \mu \mathrm{L}$ of Alamar blue substrate $\left(6.5 \mathrm{mg} / \mathrm{mL}\right.$ in PBS) was added and incubated at $37^{\circ} \mathrm{C}$ for $4 \mathrm{~h}$ in the dark condition. Cell viability is directly proportional to reduction of Alamar blue into pink-coloured solution and fluorescence intensity of solution was measured at wavelengths of $590 \mathrm{~nm}$ for emission and $560 \mathrm{~nm}$ for excitation ${ }^{21}$.

EPS quantification assay. For EPS quantification, AB cultures $(100 \mathrm{~mL})$ were grown in the absence and presence of myrtenol $(200 \mu \mathrm{g} / \mathrm{mL})$ for $24 \mathrm{~h}$ at $37^{\circ} \mathrm{C}$. To extract both cell bound and secreted EPS, AB cultures were centrifuged at $10,000 \mathrm{rpm}$ for $15 \mathrm{~min}$ to collect cell pellets and cell free culture supernatant (CFCS). The cell pellets from control and myrtenol treated samples were suspended in equal volume of isotonic buffer which contains $10 \mathrm{mM}$ Tris/ $\mathrm{HCl} \mathrm{pH} \mathrm{8.0,10} \mathrm{mM} \mathrm{EDTA,} \mathrm{2.5 \%} \mathrm{NaCl.} \mathrm{Samples} \mathrm{were} \mathrm{incubated} \mathrm{at} 4{ }^{\circ} \mathrm{C}$ for $16 \mathrm{~h}$ and centrifuged at $8000 \mathrm{rpm}$ for $10 \mathrm{~min}$ to collect cell bound EPS in supernatant which was mixed with already collected CFCS. Then, one volume of CFCS containing cell bound and secreted EPS was added with three volume of ice cold ethanol and kept at $-20^{\circ} \mathrm{C}$ overnight. After incubation, the suspension was centrifuged at $12,000 \mathrm{rpm}$ at $4^{\circ} \mathrm{C}$ for $15 \mathrm{~min}$ to collect precipitated carbohydrates as pellet. Then, the pellet was suspended in $70 \%$ ethanol and 


\begin{tabular}{|c|c|c|}
\hline Genes & Forward primer & Reverse primer \\
\hline$b f m R$ & 5'-CTGGTAGGTAATGCAGTTCG-3' & 5'-GAGAGACCCAAACCATAACC-3' \\
\hline$b a p$ & 5'-GTACTCCAGCAACGGTTGTA-3' & 5'-GAAGGATCTGCTGTATTCCA-3 \\
\hline $\operatorname{csu} A / B$ & 5'-ATGCGGTAAATACTCAAGCA-3' & 5'-TCACAGAAATATTGCCACCT-3' \\
\hline ompA & 5'-CTCTTGCTGGCTTAAACGTA-3' & 5'-GCAATTTCTGGCTTGTATTG-3' \\
\hline$p g a A$ & 5'-CACATGGCAAAAAGATGAAT-3' & 5'-CGTAGAAACCTCGAACAGTG-3' \\
\hline$p g a C$ & 5'-CAGTGGTATGGCGTGATATT-3' & 5'-GGTACTGCAACAACACTGGT-3' \\
\hline katE & 5'-GTGTCCGGTTCAGGTTTTAC-3' & 5'-GGATTCTTGACAGACCCAAC-3' \\
\hline rplB & 5'-GGTCGTAATAACAACGGTCA-3' & 5'-AATAATGCAATATGCGCTGT-3' \\
\hline
\end{tabular}

Table 3. List of primers used for qPCR analysis.

dried under vacuum. At last, the dried EPS was collected as powder and weighed. Percentage of EPS inhibition was calculated by the following formula ${ }^{51}$ EPS = Control weight - Treated weight $/$ Control weight $] \times 100$.

Microbial adhesion to hydrocarbons (MATH) assay. The influence of myrtenol on CSH of $\mathrm{AB}$ was analyzed by MATH assay ${ }^{51}$. Briefly, AB strains were grown in the absence and presence of myrtenol $(50,100$ and $200 \mu \mathrm{g} / \mathrm{mL}$ ) and cells were collected, washed with PBS and resuspended in the same. The cell suspensions were adjusted to OD of 0.9 at $600 \mathrm{~nm}$ to which $1 \mathrm{~mL}$ of toluene was added, vortexed for 2 min and kept at $4{ }^{\circ} \mathrm{C}$ for $24 \mathrm{~h}$ to allow the separation of two phases. After the separation, the OD of the aqueous phase was measured at $600 \mathrm{~nm}$ and $\mathrm{CSH}$ was calculated by the following formula: $\mathrm{CSH}(\%)=\left[1-\left(\right.\right.$ after vortex $\mathrm{OD}_{600 \mathrm{~nm}} /$ before vortex $\left.\left.\mathrm{OD}_{600 \mathrm{~nm}}\right)\right] \times 100$.

Motility assay. Swarming and twitching motility assays were performed to evaluate the effect of myrtenol on the motility of AB strains. For swarming motility assay, $0.5 \%$ of TSA plates were prepared without and with myrtenol at increasing concentrations $(50,100$ and $200 \mu \mathrm{g} / \mathrm{mL})$ and $5 \mu \mathrm{L}$ of overnight culture was introduced into the middle region of agar plates and incubated for $72 \mathrm{~h}$ at $37^{\circ} \mathrm{C}$. After incubation, motility zone was observed and measured. For twitching motility, overnight culture was stabbed on centre to till bottom of $1 \%$ TSA plate using sterile toothpick and incubated at $37^{\circ} \mathrm{C}$ for $72 \mathrm{~h}$. Then, agar was discarded carefully and plates were washed by PBS and stained with $0.4 \%$ crystal violet solution ${ }^{52}$.

$\mathrm{H}_{2} \mathrm{O}_{2}$ sensitivity assay. The influence of myrtenol treatment on the sensitivity of $\mathrm{AB}$ strains to $\mathrm{H}_{2} \mathrm{O}_{2}$ was assessed. Briefly, AB was allowed to grow in the absence and presence of myrtenol $(200 \mu \mathrm{g} / \mathrm{mL})$ for $24 \mathrm{~h}$ at $37^{\circ} \mathrm{C}$ and cells were harvested by centrifuging $8000 \mathrm{rpm}$ for $10 \mathrm{~min}$. Then, cells were resuspended in PBS containing $10 \mathrm{mM} \mathrm{H}_{2} \mathrm{O}_{2}$ and incubated for $1 \mathrm{~h}$ and surviving colonies were enumerated by serial dilution method. Also, the disc diffusion assay was performed by preparing Muller-Hinton agar (MHA) plates supplemented without and with myrtenol $(200 \mu \mathrm{g} / \mathrm{mL})$ and $\mathrm{AB}$ culture was swabbed on the surface of MHA plates. Then, sterile discs placed on the centre of plates and loaded with $10 \mu \mathrm{L}$ of $\mathrm{H}_{2} \mathrm{O}_{2}$ solution and incubated at $37^{\circ} \mathrm{C}$ for $24 \mathrm{~h}$. The diameter of the zone of clearance was observed and measured ${ }^{48}$.

Quantitative real-time PCR analysis. Total RNA from control and myrtenol $(200 \mu \mathrm{g} / \mathrm{mL})$ treated AB cultures was isolated by Trizol method and reverse transcribed into cDNA using High-capacity cDNA Reverse Transcription kit (Applied Biosystems, USA) as per the manufacturer's instructions. qPCR analysis was performed on real time PCR (7500 Sequence Detection System, Applied Biosystems Inc. Foster, CA, USA) for biofilm associated virulence genes such as $b f m R, b a p, c s u A / B$, ompA, pgaA, pgaC, katE and $r p l B$ using PCR mix (SYBR Green kit, Applied Biosystems, United States) at a predefined ratio. The primers of candidate genes are listed in Table 3. Expression of tested genes was normalized with housekeeping gene $(r p l B)$ and fold change in gene expression was calculated using $2^{(-\Delta \Delta \mathrm{Ct})} \operatorname{method}^{53}$.

Antibiotic sensitivity assay. To determine the effect of myrtenol in enhancing the susceptibility of $A B$ to antibiotics, MIC of antibiotics (amikacin, ciprofloxacin, gentamycin and trimethoprim, HiMedia, India) was determined by performing microbroth dilution assay.

Further, combinatorial efficacy of myrtenol with the antibiotics was assessed by growing $\mathrm{AB}$ strains in the absence and presence of antibiotics at $1 \times, 0.5 \times$ and $0.25 \times$ of MIC. Briefly, $200 \mu \mathrm{L}$ of TSBSY broth was added to each wells of 96 well plate and $1 \%$ overnight culture of $A B$ strains was added and treated without and with $200 \mu \mathrm{g} / \mathrm{mL}$ of myrtenol and various concentrations of antibiotics. Plates were further incubated at $37^{\circ} \mathrm{C}$ for $24 \mathrm{~h}$ and read at $600 \mathrm{~nm}$.

The disc diffusion assay was also performed as per the Clinical and Laboratory Standards Institute guidelines to determine the antibiotic sensitivity of $A B$ in the presence of myrtenol ${ }^{54}$. Briefly, TSA plates were prepared without and with myrtenol $(200 \mu \mathrm{g} / \mathrm{mL})$. Overnight culture of AB strains were swabbed on the TSA plates and sterile discs were placed on the centre of plates and loaded with MIC of antibiotics such as amikacin, ciprofloxacin, gentamycin and trimethoprim were. Then, plates were incubated at $37^{\circ} \mathrm{C}$ for $24 \mathrm{~h}$ and the zone of inhibition was measured. 
Statistical analysis. All the experiments were done in three biological replicates with three technical replicates. Data were expressed as mean \pm standard deviation (SD) and statistical significance was analyzed by oneway ANOVA method and Duncan's post hoc test using SPSS 17.0 software package (SPSS Inc., Chicago, IL). $p<0.05$ was fixed as significant value.

Received: 27 May 2020; Accepted: 26 November 2020

Published online: 15 December 2020

\section{References}

1. Wang, J., Niu, H., Wang, R. \& Cai, Y. Safety and efficacy of colistin alone or in combination in adults with Acinetobacter baumannii infection: A systematic review and meta-analysis. Int. J. Antimicrob. Agents 53, 383-400 (2019).

2. World Health Organization (WHO). Global Priority List of Antibiotic-Resistant Bacteria to Guide Research, Discovery, and Development of New Antibiotics. https://www.who.int/medicines/publications/WHO-PPL-Short_Summary_25Feb-ET_NM_WHO.pdf (2019).

3. Maragakis, L. L. \& Perl, T. M. Acinetobacter baumannii: epidemiology, antimicrobial resistance, and treatment options. Clin. Infect. Dis. Off. Publ. Infect. Dis. Soc. Am. 46, 1254-1263 (2008).

4. Morris, F. C., Dexter, C., Kostoulias, X., Uddin, M. I. \& Peleg, A. Y. The Mechanisms of disease caused by Acinetobacter baumannii. Front. Microbiol. 10, 1601 (2019).

5. Harding, C. M., Hennon, S. W. \& Feldman, M. F. Uncovering the mechanisms of Acinetobacter baumannii virulence. Nat. Rev. Microbiol. 16, 91-102 (2018).

6. Flemming, H.-C. et al. Biofilms: An emergent form of bacterial life. Nat. Rev. Microbiol. 14, 563-575 (2016).

7. AmalaReena, A., Subramaniyan, A. \& Kanungo, R. Biofilm formation as a virulence factor of Acinetobacter baumannii: An emerging pathogen in critical care units. J. Curr. Res. Sci. Med. 3, 74-78 (2017).

8. Eze, E. C., Chenia, H. Y. \& El Zowalaty, M. E. Acinetobacter baumannii biofilms: Effects of physicochemical factors, virulence, antibiotic resistance determinants, gene regulation, and future antimicrobial treatments. Infect. Drug Resist. 11, 2277-2299 (2018).

9. Koo, H., Allan, R. N., Howlin, R. P., Stoodley, P. \& Hall-Stoodley, L. Targeting microbial biofilms: Current and prospective therapeutic strategies. Nat. Rev. Microbiol. 15, 740-755 (2017).

10. Gopal, R. et al. Synergistic effects and antibiofilm properties of chimeric peptides against multidrug-resistant Acinetobacter baumannii strains. Antimicrob. Agents Chemother. 58, 1622-1629 (2014).

11. Raorane, C. J. et al. Antibiofilm and Antivirulence efficacies of flavonoids and curcumin against Acinetobacter baumannii. Front. Microbiol. 10, 990 (2019).

12. Khadke, S. K., Lee, J.-H., Woo, J.-T. \& Lee, J. inhibitory effects of honokiol and magnolol on biofilm formation by Acinetobacter baumannii. Biotechnol. Bioprocess Eng. 24, 359-365 (2019).

13. Kim, M. K. et al. Antibacterial and antibiofilm activity and mode of action of magainin 2 against drug-resistant Acinetobacter baumannii. Int. J. Mol. Sci. 19, 66 (2018).

14. Coté, H., Boucher, M.-A., Pichette, A. \& Legault, J. Anti-Inflammatory, antioxidant, antibiotic, and cytotoxic activities of Tanacetum vulgare L. Essential oil and its constituents. Med. Basel Switzerland 4, 66 (2017).

15. Lawal, O. A. \& Oyedeji, A. O. Chemical composition of the essential oils of Cyperus rotundus L. from South Africa. Molecules 14, 2909-2917 (2009).

16. Moreira, M. R. C. et al. Anxiolytic-like effects and mechanism of (-)-myrtenol: A monoterpene alcohol. Neurosci. Lett. 579, 119-124 (2014).

17. Zhao, M. et al. Presence and roles of myrtenol, myrtanol and myrtenal in Dendroctonus armandi (Coleoptera: Curculionidae: Scolytinae) and Pinus armandi (Pinales: Pinaceae: Pinoideae). Pest Manag. Sci. 76, 188-197 (2020).

18. Bhatia, S. P., McGinty, D., Letizia, C. S. \& Api, A. M. Fragrance material review on myrtenol. Food Chem. Toxicol. Int. J. Publ. Br. Ind. Biol. Res. Assoc. 6(Suppl 1), 237-40 (2008).

19. Kaufmann, D., Dogra, A. K. \& Wink, M. Myrtenal inhibits acetylcholinesterase, a known Alzheimer target. J. Pharm. Pharmacol. 63, 1368-1371 (2011).

20. Ngan, L. T. M., Moon, J.-K., Kim, J.-H., Shibamoto, T. \& Ahn, Y.-J. Growth-inhibiting effects of Paeonia lactiflora root steam distillate constituents and structurally related compounds on human intestinal bacteria. World J. Microbiol. Biotechnol. 28, 1575-1583 (2012).

21. Selvaraj, A., Jayasree, T., Valliammai, A. \& Pandian, S. K. Myrtenol attenuates MRSA biofilm and virulence by suppressing sarA expression dynamism. Front. Microbiol. 10, 1-15 (2019).

22. Viana, A. F. S. C. et al. (-)-Myrtenol accelerates healing of acetic acid-induced gastric ulcers in rats and in human gastric adenocarcinoma cells. Eur. J. Pharmacol. 854, 139-148 (2019).

23. Davenport, E. K., Call, D. R. \& Beyenal, H. Differential protection from tobramycin by extracellular polymeric substances from Acinetobacter baumannii and Staphylococcus aureus biofilms. Antimicrob. Agents Chemother. 58, 4755-4761 (2014).

24. Limoli, D. H., Jones, C. J. \& Wozniak, D. J. Bacterial extracellular polysaccharides in biofilm formation and function. Microbiol. Spectr. 3, MB-0011-2014 (2015).

25. Vu, B., Chen, M., Crawford, R. J. \& Ivanova, E. P. Bacterial extracellular polysaccharides involved in biofilm formation. Molecules 14, 2535-2554 (2009).

26. Dertli, E., Mayer, M. J. \& Narbad, A. Impact of the exopolysaccharide layer on biofilms, adhesion and resistance to stress in Lactobacillus johnsonii FI9785. BMC Microbiol. 15, 8 (2015).

27. May, H. C. et al. Thioredoxin modulates cell surface hydrophobicity in Acinetobacter baumannii. Front. Microbiol. 10, 2849 (2019).

28. Nait Chabane, Y. et al. Virstatin inhibits biofilm formation and motility of Acinetobacter baumannii. BMC Microbiol. 14, 62 (2014).

29. Skiebe, E. et al. Surface-associated motility, a common trait of clinical isolates of Acinetobacter baumannii, depends on 1,3-diaminopropane. Int. J. Med. Microbiol. 302, 117-128 (2012).

30. Wood, C. R., Ohneck, E. J., Edelmann, R. E. \& Actis, L. A. A Light-regulated Type I pilus contributes to Acinetobacter baumannii biofilm, motility, and virulence functions. Infect. Immun. 86, 66 (2018).

31. Clemmer, K. M., Bonomo, R. A. \& Rather, P. N. Genetic analysis of surface motility in Acinetobacter baumannii. Microbiology 157, 2534-2544 (2011).

32. Nait Chabane, Y. et al. Characterisation of pellicles formed by Acinetobacter baumannii at the air-liquid interface. PLoS ONE 9, e111660 (2014).

33. Bhargava, N., Sharma, P. \& Capalash, N. Pyocyanin stimulates quorum sensing-mediated tolerance to oxidative stress and increases persister cell populations in Acinetobacter baumannii. Infect. Immun. 82, 3417-3425 (2014).

34. Ramanathan, S. et al. Biofilm inhibitory efficiency of phytol in combination with cefotaxime against nosocomial pathogen Acinetobacter baumannii. J. Appl. Microbiol. 125, 56-71 (2018). 
35. Sivaranjani, M., Srinivasan, R., Aravindraja, C., Karutha Pandian, S. \& Veera Ravi, A. Inhibitory effect of $\alpha$-mangostin on Acinetobacter baumannii biofilms-An in vitro study. Biofouling 34, 579-593 (2018).

36. Draughn, G. L. et al. The Structure of the biofilm-controlling response regulator BfmR from Acinetobacter baumannii reveals details of its DNA-binding mechanism. J. Mol. Biol. 430, 806-821 (2018).

37. Russo, T. A. et al. The response regulator BfmR is a potential drug target for Acinetobacter baumannii. eSphere 1, e00082-16 (2016).

38. Brossard, K. A. \& Campagnari, A. A. The Acinetobacter baumannii biofilm-associated protein plays a role in adherence to human epithelial cells. Infect. Immun. 80, 228-233 (2012).

39. Loehfelm, T. W., Luke, N. R. \& Campagnari, A. A. Identification and characterization of an Acinetobacter baumannii biofilmassociated protein. J. Bacteriol. 190, 1036-1044 (2008).

40. Gaddy, J. A., Tomaras, A. P. \& Actis, L. A. The Acinetobacter baumannii 19606 OmpA protein plays a role in biofilm formation on abiotic surfaces and in the interaction of this pathogen with eukaryotic cells. Infect. Immun. 77, 3150-3160 (2009).

41. Choi, A. H. K., Slamti, L., Avci, F. Y., Pier, G. B. \& Maira-Litrán, T. The pgaABCD locus of Acinetobacter baumannii encodes the production of poly-beta-1-6- $N$-acetylglucosamine, which is critical for biofilm formation. J. Bacteriol. 191, 5953-5963 (2009).

42. Ajiboye, T. O. Contributions of reactive oxygen species, oxidative DNA damage and glutathione depletion to the sensitivity of Acinetobacter baumannii to 2-(2-nitrovinyl) furan. Microb. Pathog. 128, 342-346 (2019).

43. Heindorf, M., Kadari, M., Heider, C., Skiebe, E. \& Wilharm, G. Impact of Acinetobacter baumannii superoxide dismutase on motility, virulence, oxidative stress resistance and susceptibility to antibiotics. PLoS ONE 9, e101033 (2014).

44. Gurunathan, S., Han, J. W., Kwon, D.-N. \& Kim, J.-H. Enhanced antibacterial and anti-biofilm activities of silver nanoparticles against Gram-negative and Gram-positive bacteria. Nanoscale Res. Lett. 9, 373 (2014).

45. Pemovska, T., Bigenzahn, J. W. \& Superti-Furga, G. Recent advances in combinatorial drug screening and synergy scoring. Curr. Opin. Pharmacol. 42, 102-110 (2018).

46. Valliammai, A. et al. Proteomic profiling unveils citral modulating expression of IsaA, CodY and SaeS to inhibit biofilm and virulence in methicillin-resistant Staphylococcus aureus. Int. J. Biol. Macromol. 158, 208-221 (2020).

47. Santhakumari, S. et al. In vitro and in vivo effect of 2,6-Di-tert-butyl-4-methylphenol as an antibiofilm agent against quorum sensing mediated biofilm formation of Vibrio spp. Int. J. Food Microbiol. 281, 60-71 (2018).

48. Sethupathy, S., Vigneshwari, L., Valliammai, A., Balamurugan, K. \& Pandian, S. K. 1-Ascorbyl 2,6-dipalmitate inhibits biofilm formation and virulence in methicillin-resistant Staphylococcus aureus and prevents triacylglyceride accumulation in Caenorhabditis elegans. RSC Adv. 7, 23392-23406 (2017).

49. Prasath, K. G., Sethupathy, S. \& Pandian, S. K. Proteomic analysis uncovers the modulation of ergosterol, sphingolipid and oxidative stress pathway by myristic acid impeding biofilm and virulence in Candida albicans. J. Proteomics 208, 103503 (2019).

50. Muthamil, S., Balasubramaniam, B., Balamurugan, K. \& Pandian, S. K. Synergistic effect of quinic acid derived from Syzygium cumini and undecanoic acid against Candida spp. biofilm and virulence. Front. Microbiol. 9, 2835 (2018).

51. Sivasankar, C. et al. A combination of ellagic acid and tetracycline inhibits biofilm formation and the associated virulence of Propionibacterium acnes in vitro and in vivo. Biofouling 32, 397-410 (2016).

52. Otton, L. M., da Silva Campos, M., Meneghetti, K. L. \& Corção, G. Influence of twitching and swarming motilities on biofilm formation in Pseudomonas strains. Arch. Microbiol. 199, 677-682 (2017).

53. Valliammai, A. et al. 5-Dodecanolide interferes with biofilm formation and reduces the virulence of methicillin-resistant Staphylococcus aureus (MRSA) through up regulation of agr system. Sci. Rep. 9, 13744 (2019).

54. Wayne PA. Clinical and Laboratory Standards Institute, Performance Standards for Antimicrobial Disk Susceptibility Test; Approved Standard-11th Edition, Clinical and Laboratory Standards Institute (CLSI) (2017).

\section{Acknowledgements}

Authors sincerely acknowledge the computational and bioinformatics analyses provided by the Bioinformatics Infrastructure Facility (funded by DBT, GOI; File No. BT/BI/25/012/2012, BIF). The authors also thankfully acknowledge DST-FIST [Grant No. SR/FST/LSI-639/2015(C)], UGC-SAP [Grant No. F.5-1/2018/DRS-II (SAPII)] and DST-PURSE [Grant No. SR/PURSE Phase $2 / 38(\mathrm{G})$ ] for providing instrumentation facilities. AS thanks UGC for the financial assistance in the form of a Basic Scientific Research Fellowship [Sanction No. F.25-1/201314 (BSR)/7-326/2011 (BSR)]. SKP is thankful to UGC for Mid-Career Award [F.19-225/2018 (BSR)] and RUSA 2.0 [F.24-51/2014-U, Policy (TN Multi-Gen), Dept. of Edn, GoI]. CS acknowledges the ICMR for the financial support in the form of Research Associateship [File No: 3/1/2/1/Env/2019-NCD-1].

\section{Author contributions}

S.K.P. and A.S. designed the study. A.S., G.S., M.S. and A.V. performed the experiments. A.S. analyzed the data, prepared the figures and tables, and wrote the manuscript. A.V., C.S. and S.K.P. revised the manuscript. All authors have read and approved the final version of the manuscript.

\section{Competing interests}

The authors declare no competing interests.

\section{Additional information}

Supplementary Information The online version contains supplementary material available at https://doi. org/10.1038/s41598-020-79128-X.

Correspondence and requests for materials should be addressed to S.K.P.

Reprints and permissions information is available at www.nature.com/reprints.

Publisher's note Springer Nature remains neutral with regard to jurisdictional claims in published maps and institutional affiliations. 
(c) (i) Open Access This article is licensed under a Creative Commons Attribution 4.0 International cc) License, which permits use, sharing, adaptation, distribution and reproduction in any medium or format, as long as you give appropriate credit to the original author(s) and the source, provide a link to the Creative Commons licence, and indicate if changes were made. The images or other third party material in this article are included in the article's Creative Commons licence, unless indicated otherwise in a credit line to the material. If material is not included in the article's Creative Commons licence and your intended use is not permitted by statutory regulation or exceeds the permitted use, you will need to obtain permission directly from the copyright holder. To view a copy of this licence, visit http://creativecommons.org/licenses/by/4.0/.

(C) The Author(s) 2020 